\title{
Jumping Mechanism for Asteroid Rover with the Use of Resonance and Electrical Stiffness Switching
}

\author{
By Yoshiki SugawarA ${ }^{1)}$, Kou Mizuguchi ${ }^{1)}$ and Nobuyuki KobaYASHI ${ }^{1)}$ \\ ${ }^{1)}$ Department of Mechanical Engineering, Aoyama Gakuin University, Sagamihara, Japan
}

(Received July 14th, 2009)

\begin{abstract}
It is not easy for asteroid rover with wheels to move on the surface of asteroids because such an astral body has two special features. One feature is that there is no air and it induces vacuum metalizing of metal slide components. The other feature is that their quite small gravity induces bad controllability of rover with wheels which requires enough frictional force between ground and wheels. Therefore, it is preferable for rover to use jumping mechanism without metal slide component and with low energy consumption. In this paper, a jumping mechanism is proposed and the mechanism uses a energy which is stored by resonance of flexible part. However, simply giving resonance results in low height of jumping. Therefore, electrical stiffness switching system is implemented to realize a effective jumping. Electrical stiffness switching is realized by piezoelectric element and external capacitor which is connected to them. Two method of stiffness switching are introduced. One is stiffness hardening and the other is stiffness softening which solve the problem of stiffness hardening. To validate the proposed mechanisms, numerical analyses are carried out and feasibilities of application for asteroid rover are studied.
\end{abstract}

Key Words: Rover, Jumping Mechanism, Asteroid, Piezoelectric Element, Hopping Mechanism

\begin{tabular}{|c|c|}
\hline \multicolumn{2}{|c|}{ Nomenclature } \\
\hline$L$ & : Length of the flexible element \\
\hline$h$ & : Thickness \\
\hline$w$ & : Width \\
\hline$\rho$ & : Mass density \\
\hline$I$ & : Second moment of area \\
\hline$E$ & : Young's modulus \\
\hline$A$ & : Cross sectional area \\
\hline$m$ & : Mass \\
\hline$k$ & : Stiffness of spring \\
\hline$y$ & : Vertical displacement \\
\hline$f_{r}$ & : Reaction force from ground \\
\hline$g$ & : Gravitational acceleration \\
\hline$d_{31}$ & $\begin{array}{l}\text { Piezoelectric constant of piezoelectric } \\
\text { element for } 31 \text { direction }\end{array}$ \\
\hline$\varepsilon_{33}^{T}$ & $\begin{array}{l}\text { : Permittivity of piezoelectric element for } \\
33 \text { direction }\end{array}$ \\
\hline$s_{11}^{E}$ & : Material compliance $\left(=1 / E_{p}\right)$ \\
\hline$\xi_{i}$ & : Modal coordinate of i-th mode \\
\hline$\phi_{i}$ & : Shape function of i-th mode \\
\hline$z$ & $\begin{array}{l}\text { Relative displacement with respect to } \\
\text { rigid body }\end{array}$ \\
\hline$v$ & : Applied voltage to piezoelectric element \\
\hline$q$ & : Electric charge within circuit \\
\hline$f_{a}$ & : Force generated by actuator \\
\hline$\Theta$ & : Electromechanical coupling coefficient \\
\hline Subscript & \\
\hline$b$ & : flexible beam \\
\hline$p$ & : piezoelectric element \\
\hline$t$ & : Tip mass \\
\hline$r$ & : Rigid body \\
\hline$f$ & : flexible element \\
\hline
\end{tabular}

$\begin{array}{lll}l & : & \text { Lower mass } \\ u & : & \text { Upper mass } \\ (i) & : & \text { i-th flexible component }\end{array}$

\section{Introduction}

To reveal the origin of the earth and solar system, asteroids have been gathering a lot of attentions and some explorers have been sent to some asteroids in the last couple of decades. NASA has launched NEAR Shoemaker ${ }^{1)}$ in 1997 and it approached to asteroid "Mathilde" and "Eros" and it sent a wealth of interesting data. NASA also has launched Deep Space $1^{2)}$ in 1998 and it explored asteroid "Braille" and comet "Borrelly". Furthermore, ESA, RKA and JAXA also has sent some explorers to such a asteroid. But almost all missions for small astral bodies include explorations with only remote observation and there are a very few cases to survey on their surfaces. One of the cases, Hayabusa developed by JAXA has reached to asteroid "Itokawa" in 2005 and it has sent a great deal of significant data by remote observation ${ }^{3}$. In addition to the data by remote observation, Hayabusa has carried out touch down mission and released a small rover called MINERVA ${ }^{4)}$. Such a rover on asteroid is gaining attentions and some of forthcoming missions will execute a release of rovers. However, there are not a lot of prominent achievements by rovers for asteroid and there are some technical difficulties for such a rover, it is not easy for rover to survey on the asteroid's surface.

The most significant difficulty on asteroid rover comes from smallness of the asteroid's gravity, e.g. Eros's gravity on the surface is less than $0.006\left[\mathrm{~m} / \mathrm{s}^{2}\right]$ and Itokawa has less than $0.1\left[\mathrm{~mm} / \mathrm{s}^{2}\right]$. Such a small gravity causes a small frictional force between rover and asteroid's surface. Therefore, the 
conventional locomotion method on astral bodies, i.e. wheel drives, can not work efficiently. In other words, horizontal locomotion by wheel drive yields non-horizontal components of reaction force and then the rover begins to bounce and loses its controllability. In order to solve such a difficulty, some jumping systems are proposed for astral bodies with small gravity. Forces or torques for jumping systems are generally produced by motor drive, e.g. reaction wheel. MINERVA ${ }^{4)}$ also employs a jumping locomotion which is realized by torque generated by reaction wheel. However, implementation of motor has two problems due to the environment of space explorations and they are as follows:

A) Extremely high vacuum environment induces vacuum metalizing of metal slide component in the system, e.g. bearing and shaft of a rotating system, therefore isolation from vacuum environment have to be implemented. Consequently the volume of the actuator become larger and reliability of the system become lower.

B) The energy for the system is limited due to the limitations on mounting space for batteries and solar cells. Hence, there are possibilities that desirable forces or torques can not be produced. Especially ample margin of energy is required for asteroid rover because it is difficult to obtain detail data of asteroids before arrival of the rover at them.

In other area except for space development, a lot of studies for jumping mechanism have been done. For instance, Hougen, et al. implemented a spring foot for jumping in miniature robot with rolling wheel ${ }^{5)}$ and Hosoda, et al. reinforced the performance of a biped robot by installing the jumping function as locomotion method ${ }^{6}$. However, almost all jumping mechanism can not escape abovementioned problems. Therefore, it is preferable for rovers to implement jumping mechanisms without metal slide component and with efficient energy consumption.

In this paper, two jumping mechanism is proposed and both of them utilize the energy which is stored by resonance of flexible part. Since resonance can be given by small actuator, it can be expected that small jumping mechanism with efficient energy consumption can be realized. For example, such an actuator is oscillator by electromagnetic forces. However, simple resonance by small force results in low height of jumping. To improve such a small jumping, electrical stiffness switching is implemented on both of proposed mechanisms and such a stiffness switching produces energy leap of the system which enables the improvement of the jumping height. Electrical stiffness switching is realized by piezoelectric element and external capacitor which is connected to that element and such a method has good reliability because it can be done electrically.

In the following, at first the basic principle of jumping is explained by the use of simple 2 DOF spring -mass system. In Section 3, the mechanism which hardens the stiffness is introduced and the results of numerical analyses and its features are studied. In Section 4, the mechanism which softens the stiffness is introduced to improve disadvantages of the proposed mechanism in Section 3. Furthermore numerical analyses for the model are also carried out in Section 4.
Finally the paper is summarized and some future works are denoted in Section 5.

\section{Preliminary Study on 2 DOF Spring-Mass System}

Fig. 1 shows 2 DOF spring- mass system. An upper body and a lower body are connected with each other through a spring. The lower body is the only component which has contact with ground and an actuator is mounted on the upper body. Note that any style of the actuator is not specified in the analysis. The mathematical expression of the system is represented by

$$
\left[\begin{array}{cc}
m_{l} & 0 \\
0 & m_{u}
\end{array}\right]\left[\begin{array}{l}
\ddot{y}_{l}(t) \\
\ddot{y}_{u}(t)
\end{array}\right]+\left[\begin{array}{cc}
k & -k \\
-k & k
\end{array}\right]\left[\begin{array}{l}
y_{l}(t) \\
y_{u}(t)
\end{array}\right]=\left[\begin{array}{l}
-m_{l} g+f_{r} \\
-m_{u} g+f_{a}
\end{array}\right]
$$

where $f_{r}$ is given by

$$
\begin{gathered}
f_{r}=\left\{\begin{array}{cl}
m_{l} g-k y_{u}(t) & m_{l} g-k y_{u}(t)>0 \\
0 & m_{l} g-k y_{u}(t) \leq 0
\end{array}\right. \\
\text { Gravity } \quad y_{u} \leftarrow \begin{array}{ll}
m_{u} & \text { Actuator }
\end{array} \\
\downarrow
\end{gathered}
$$

Fig. 1. 2 DOF spring- mass system.

To cause a resonant oscillation of the system, $f_{a}$ is given by

$$
f_{a}=f_{0} \cos \omega_{u} t
$$

where $\omega_{u}=\sqrt{k / m_{u}}$ and $f_{0}$ is the amplitude of $f_{a}$. Suppose $t_{\text {hop }}$ is the time when the lower body takes off from ground. Then, $y_{u}(t)$ for $t<t_{\text {hop }}$ is given by ${ }^{9)}$

$$
y_{u}(t)=\frac{f_{0} \omega_{u} t}{2 k} \cos \omega_{u} t-\frac{m_{u} g}{k} \quad \text { for } \quad 0 \leq t<t_{\text {hop }} .
$$

Eq. (4) indicates that the amplitude of $y_{u}(t)$ grows gradually since the exciting force is supposed to be small as stated above. When the amplitude reaches to a certain level, second condition of Eq. (2) is satisfied and the lower body takes from ground. Suppose $y_{u 0}$ is the displacement of the lower body at local minimum point right before the lower body's takeoff. Then, the trajectory of the center of mass after the takeoff is represented by

$$
\begin{array}{r}
y_{G \max } \approx \frac{m_{u}^{2}}{2 g\left(m_{l}+m_{u}\right)^{2}}\left(\frac{k y_{u 0}^{2}}{m_{u}}-\frac{m_{l}^{2} g^{2}}{m_{u} k}-\frac{2 m_{l} g^{2}}{k}+2 g y_{u 0}\right) \\
+\frac{m_{u}}{m_{l}+m_{u}}\left(\frac{m_{l} g}{k}+l\right)
\end{array}
$$

Since energy is increased gradually by small force, the energy at $t=t_{\text {hop }}$ is almost minimum amount required for the takeoff of the lower body. Therefore, higher jumping can not be expected as long as monotonic increase of energy by small force is applied. Furthermore, $y_{u 0}$ is calculated as

$$
y_{u 0} \approx-\frac{m_{l}+2 m_{u}}{k} g
$$

and it indicates that smaller value of $g$ results in smaller compression of the spring. Then, the system takes off before enough storage of energy and consequently higher jumping 
can not be expected even in smaller gravity circumstance. Therefore, some additional operation and mechanism is required to utilize the energy stored by resonance for jumping.

\section{Jumping Mechanism with Stiffness Hardening}

\subsection{Stiffness hardening before takeoff}

Differentiation of Eq. (5) with respect to $k$ yields

$$
\frac{\partial y_{G \max }}{\partial k}>0 \text {. }
$$

Eq. (7) indicates that switching to hard spring at minimum local point right before the jumping results in the increase of the reachable height of the center of mass. Note that an implementation of hard spring as original system does not lead to improvement of the jumping height because the depth of compression of the spring does not become deeper before takeoff of the lower body. Therefore, it is important to switch the stiffness right before the jumping.

\subsection{Electrical stiffness switching method}

Suppose the system as shown in Fig. 2 and the system consists of a flexible beam, a tip mass, a piezoelectric element and an external capacitor. One end of the flexible beam is fixed and the tip mass is attached to the other end of the beam. The piezoelectric element is attached on the beam and the external capacitor is connected to the element.

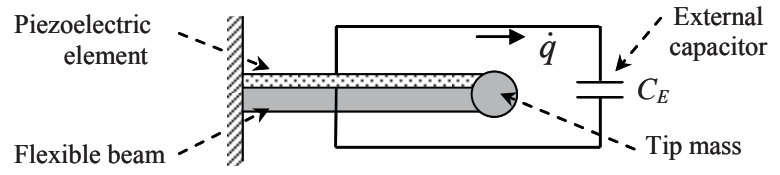

Fig. 2. Flexible beam with piezoelectric element and external circuit.

The mathematical expression about the system is given by ${ }^{7)}$

$$
\begin{aligned}
& m \ddot{\xi}_{i}+k \xi_{i}-\Theta v=0 \\
& \Theta \xi_{i}+C_{P}^{S} v=q .
\end{aligned}
$$

Taking the voltage drop at external capacitor into account, Eq. (8) and Eq. (9) yields the following expression as

$$
m \ddot{\xi}_{i}+\left(k+\frac{\Theta^{2}}{C_{P}^{S}+C_{E}}\right) \xi_{i}=0 .
$$

Eq. (10) indicates that Eq. (8) and Eq. (9) can be represented by an equivalent mechanical model and that the stiffness of the mechanical system can be changed by electrical method. Smaller capacitance of the capacitor realizes the larger stiffness of the system. Illustration of Eq. (10) is shown in Fig. 3. Note that the mass and equivalent spring in Fig. 3 has same structure of the upper mass and the spring in Fig. 1. Therefore, it can be expected that the system in Fig. 3 can be used to realize the stiffness switching mentioned in Subsection 3.1.

\subsection{Switching of capacitance}

As discussed in Subsection 3.2, switching of capacitance is significant in stiffness switching. In this subsection, the meaning of switching of capacitance is shown by the observation of energy change before and after the switching. Suppose $C_{E 1}$ and $C_{E 2}$ are the capacitance of the external capacitors. Then, the change of the potential energy $\Delta U_{p}$ is denoted as

$$
\Delta U_{p}=\frac{1}{2} \frac{\Theta^{2}}{\bar{C}_{2}} \xi_{i}^{2}-\frac{1}{2} \frac{\Theta^{2}}{\bar{C}_{1}} \xi_{i}^{2}=\frac{1}{2} \frac{q^{2}}{\bar{C}_{2}}-\frac{1}{2} \frac{q^{2}}{\bar{C}_{1}}
$$

where $\quad \bar{C}_{1}=C_{P}^{S}+C_{1}, \quad \bar{C}_{2}=C_{P}^{S}+C_{2} \quad$ and the relation $q=\Theta \xi_{i}^{28)}$ is applied to the transformation of Eq. (11). Note that Eq. (11) indicates that the capacitance should be changed under the condition that the electric charge within the circuit is kept in same value. For example the circuit shown in Fig. 4 can realize such a switching of capacitance.

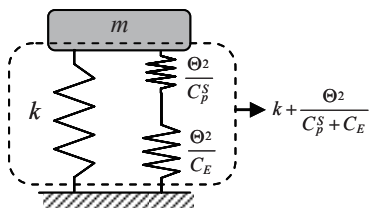

Fig. 3. Equivalent mechanical model of the system shown in Fig. 2.

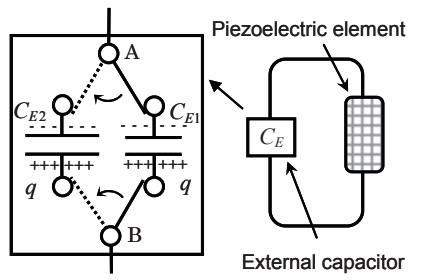

Fig. 4. Example of external capacitor.

Fig. 4 shows that $C_{E 1}$ is inserted between point $\mathrm{A}$ and $\mathrm{B}$ at first and $C_{E 2}$ with electric charge $q$ is inserted between point A and B after capacitance switching. Such a switch can be done by electrical method by the use of FET and so on, therefore reliable switching is possible.

3.4. Proposed jumping mechanism by stiffness hardening

Suppose the system as shown in Fig. 5. The system consists of a rigid body, a tip mass and a flexible element. Furthermore, the flexible element consists of a flexible beam and a piezoelectric element and an external capacitor as shown in the right figure of Fig. 5. For the sake of convenience, the component which consists of the flexible element, the tip mass and the actuator is called "excited component" in the later.

To derive the mathematical expression of the system, following assumptions are introduced.

- The flexible element has uniform shape and uniform material property.

- The collision between ground and rigid body is completely inelastic.

- The deformation of the beam does not have influence on the rotational motion of the system and only vertical translational motion is considered for deriving the mathematical model.

- Only bending deformation and the first mode deformation are supposed for flexible beam, i.e. $z_{f}(x, t)=\phi_{1}(x) \xi_{1}(t)$.

- Internal viscosity of flexible element is omitted.

Then the mathematical expression is given by

$$
M \ddot{X}+\hat{K} X=F
$$

where

$$
X=\left[\begin{array}{l}
y_{r}(t) \\
\xi_{1}(t)
\end{array}\right], \quad F=\left[\begin{array}{c}
f_{r}+f_{a}-M_{G 1} g \\
\phi(L) f_{a}-M_{G 2} g
\end{array}\right],
$$




$$
M=\left[\begin{array}{ll}
M_{11} & M_{12} \\
M_{21} & M_{22}
\end{array}\right], \hat{K}=\left[\begin{array}{cc}
0 & 0 \\
0 & \hat{K}_{22}
\end{array}\right]=\left[\begin{array}{cc}
0 & 0 \\
0 & K_{22}+\frac{\Theta^{2}}{C_{p}^{S}+C_{E}}
\end{array}\right]
$$

and the details of $M_{11}, M_{12}, M_{21}, M_{22}, K_{22}, \Theta_{c}, C_{p}^{S}$, $M_{G 1}$ and $M_{G 2}$ are shown in the appendix.

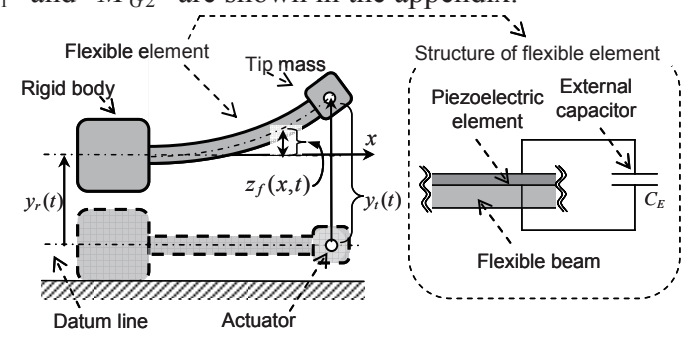

Fig. 5. Jumping mechanism with stiffness switching.

Introducing the absolute coordinate of the tip mass as $y_{t}(t)=y_{r}(t)+z_{f}(L, t)$ and transformation matrix $\Omega$ which satisfies $X=\Omega \bar{X}$ for $\bar{X}=\left[\begin{array}{ll}y_{r}(t) & y_{t}(t)\end{array}\right]^{T}$, Eq. (12) is rewritten as

$$
\bar{M} \ddot{\bar{X}}+\bar{K} \bar{X}=\bar{F}
$$

where $\bar{M}=\Omega^{T} M \Omega, \quad \bar{K}=\Omega^{T} \hat{K} \Omega, \quad \bar{F}=\Omega^{T} F$. Note that $\bar{M}, \bar{K}$ and $\bar{F}$ can be approximated as

$$
\begin{gathered}
\bar{M}=\left[\begin{array}{cc}
m_{r} & 0 \\
0 & m_{t}
\end{array}\right], \quad \bar{F}=\left[\begin{array}{l}
f_{r}-m_{r} g \\
f_{a}-m_{t} g
\end{array}\right], \\
\bar{K}=\left[\begin{array}{cc}
\hat{K}_{22} / \phi_{1}(L)^{2} & -\hat{K}_{22} / \phi_{1}(L)^{2} \\
-\hat{K}_{22} / \phi_{1}(L)^{2} & \hat{K}_{22} / \phi_{1}(L)^{2}
\end{array}\right]
\end{gathered}
$$

when the flexible element has thin and long shape, which is reasonable shape to cause resonant oscillation. Then, the system becomes structurally analogous with the system shown in Fig. 1. Therefore, the jumping procedure discussed in Subsection 3.1 becomes possible.

\subsection{Jumping mechanism by stiffness hardening with multiple excited component}

When the capacitor is switched to harden the stiffness, large voltage may be generated because capacitance become small and electric charge within the circuit is kept. Hence, to prevent such a large voltage, the system shown in Fig. 6 is introduced.

The system shown in Fig. 6 consists of a rigid body, $N$ tip masses, $N$ flexible elements and $N$ actuators. In other word, the system consists of the rigid body and $N$ excited components introduced in Fig. 5. Each excited component has same structure with that of the system shown in Fig. 5. Each flexible part and tip mass has same physical parameters with each other. It is easy to guess that synchronous exciting of flexible elements by their actuators and simultaneous stiffness hardening can cause the same jumping procedure with that denoted in Subsection 3.3. Consequently, new parameter $N$ is introduced and degree-of-freedom for the parameter decision is increased.

Introducing the displacement of $\mathrm{i}$-th tip mass $u_{(i)}(L, t)=\phi_{1}(L) \xi_{1(i)}(t)$ and the force generated by the i-th actuator $f_{a(i)}$, the mathematical expression of the system is given by

where

$$
M_{N} \ddot{X}_{N}+\hat{K}_{N} X_{N}=F_{N}
$$

$$
\begin{aligned}
& X_{N}=\left[\begin{array}{lllll}
y_{r}(t) & \xi_{1(1)}(t) & \xi_{1(2)}(t) & \cdots & \xi_{1(N)}(t)
\end{array}\right]^{T} \\
& M_{N}=\left[\begin{array}{ll}
M_{11 N} & M_{12 N} \\
M_{12 N}^{T} & M_{22 N}
\end{array}\right], \hat{K}_{N}=\left[\begin{array}{cc}
0 & 0_{1 \times N} \\
0_{1 \times N} & \hat{K}_{22 N}
\end{array}\right], \\
& F_{N}=\left[\begin{array}{c}
f_{r}+\sum_{i=1}^{N} f_{a(i)}-M_{G 1} g \\
\phi(L) f_{a(1)}-M_{G 2} g \\
\vdots \\
\phi(L) f_{a(N)}-M_{G 2} g
\end{array}\right] \in \mathfrak{R}(N+1) \times 1, \\
& M_{11 N}=M_{11} \in \mathfrak{R}^{1 \times 1} \text {, } \\
& M_{12 N}=\left[\begin{array}{lll}
M_{12} & \cdots & M_{12}
\end{array}\right] \in \mathfrak{R}^{1 \times N} \text {, } \\
& M_{22 N}=\operatorname{diag}\left(\begin{array}{lll}
M_{22} & \cdots & M_{22}
\end{array}\right) \in \mathfrak{R}^{N \times N}, \\
& \hat{K}_{22 N}=\operatorname{diag}\left(\begin{array}{lll}
\hat{K}_{22} & \cdots & \hat{K}_{22}
\end{array}\right) \in \mathfrak{R}^{N \times N} .
\end{aligned}
$$

Note that $M_{11}, M_{12}, M_{21}, M_{22}, \hat{K}_{22}, M_{G 1}$ and $M_{G 2}$ are same with those used in Eq. (12)

To cause synchronous resonant oscillation of all excited components, the excited forces by the actuators are defined as

$$
f_{a(i)}=f_{0} \cos \omega_{f} t \text { for } i=1,2, \cdots N
$$

where $\omega_{f}=\sqrt{\hat{K}_{22} / M_{22}}$.

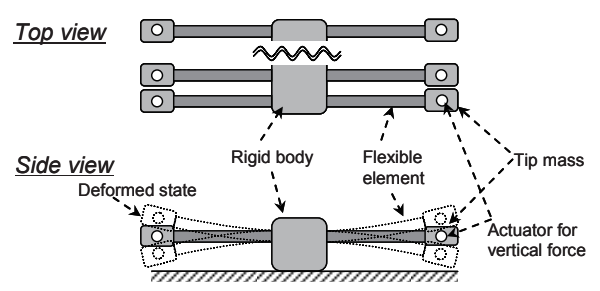

Fig. 6. Jumping mechanism with multiple flexible element.

\subsection{Numerical analysis and discussions}

To validate the proposed method, numerical analysis is carried out about the parameter shown in Table 1. Note that the parameters about piezoelectric element are reference values about "C-91 piezoelectric ceramic" produced by Fuji Ceramics Corporation ${ }^{10)}$.

Table 1. Parameters for analysis in the case of stiffness hardening.

\begin{tabular}{|l|l|}
\hline$N=100$ & $h_{b}=0.1[\mathrm{~mm}]$ \\
\hline$L=0.06[\mathrm{~m}]$ & $h_{p}=0.5[\mathrm{~mm}]$ \\
\hline$w_{b}=w_{p}=0.5[\mathrm{~mm}]$ & $g=9.81\left[\mathrm{~m} / \mathrm{s}^{2}\right]$ \\
\hline$m_{b}=5.0[\mathrm{~g}]$ & $\rho_{b}=2.7 \times 10^{3}\left[\mathrm{~kg} / \mathrm{m}^{3}\right]$ \\
\hline$m_{t}=0.1[\mathrm{~g}]$ & $\rho_{p}=7.7 \times 10^{3}\left[\mathrm{~kg} / \mathrm{m}^{3}\right]$ \\
\hline$E_{b}=7.0 \times 10^{10}[\mathrm{~Pa}]$ & $C_{E 1}=1.0 \times 10^{-7}[\mathrm{~F}]$ \\
\hline$E_{p}=5.9 \times 10^{10}[\mathrm{~Pa}]$ & $C_{E 2}=1.0 \times 10^{-9}[\mathrm{~F}]$ \\
\hline$\varepsilon_{33}^{T}=5500$ & $d_{31}=-330 \times 10^{-12}[\mathrm{~m} / \mathrm{V}]$ \\
\hline
\end{tabular}

The results of numerical analyses are shown in Fig.7. The upper, middle and lower figure of Fig.7 indicate the time history of rigid body $y_{r}(t)$, the tip mass of the 1st excited component $y_{u(1)}(t)$ where $y_{t(1)}(t)=y_{r}(t)+\phi_{1}(L) z_{u(1)}(t)$ and center of the mass $\gamma(t)$. The trajectories of the tip mass of the other excited component, i.e. $y_{u(i)}(t)$ for $i=2,3, \cdots, N$ are same with that of the 1 st because all components are excited synchronously. Therefore, only the time history of $y_{t(1)}(t)$ is shown in Fig. 7. The results given by solid line and 
dashed line correspond to the numerical analysis of the proposed jumping mechanism with and without stiffness hardening respectively. The stiffness hardening is carried out at $0.365[\mathrm{sec}$.$] and the external capacitance is switched from$ $C_{E 1}$ to $C_{E 2}$ at that time.

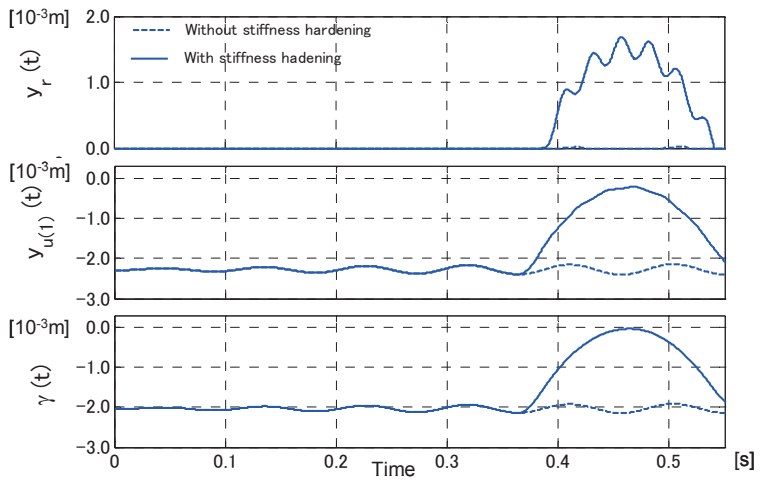

Fig. 7. Numerical analysis of the jumping mechanisms shown in Fig. 6.

As is clear from Fig. 7, achieved maximum heights of the rigid body and the center of mass in the case with stiffness switching are much higher than those in the case without stiffness switching. Therefore, it can be said the proposed jumping mechanism is effective jumping mechanism. However, it is difficult to say that the achieved maximum height of the proposed mechanism is absolutely enough. Brief parameter study indicates that the relation of each parameter is quite complex and it is not easy to improve the reachable maximum height of the jumping mechanism because it is difficult to achieve high increasing rate of stiffness by practical parameters. Furthermore, features of such a proposed mechanism can come down to the studies shown in Section 2 and they shows that compressed displacement of the spring (in the case of the system shown in Fig.6, the bending deformation of the beam) does not grow deeply when the gravitational acceleration becomes small, which is indicated by Eq. (6). Therefore, it is difficult to expect some applications of the proposed mechanism to asteroid rovers. However, there may be some possibilities of their applications if other functional materials are used for stiffness switching instead of piezoelectric element, e.g. Piezoelectric elastomer.

\section{Jumping Mechanism with the Use of Electrical Stiffness Softening}

\subsection{Excitation method without change of reaction force}

As denoted in Subsection 3.6, the jumping mechanism proposed in Section 3 shows disadvantage in smaller gravity. To overcome such a disadvantage, following exciting forces by the actuators of the jumping mechanism shown in Fig. 6 are introduces as

$$
f_{a(i)}=\left\{\begin{array}{rll}
f_{0} \cos \omega_{f} & \text { for } \quad i=1,3,5, \cdots 2 n-1 \\
-f_{0} \cos \omega_{f} & \text { for } \quad i=2,4,6 \cdots 2 n
\end{array}\right.
$$

where $2 n$ is the number of excited component and $N=2 n$, that is, $N$ is set to be even number. Eq. (16) indicates that the displacements of half of the excited components are opposite to the displacements of the other half of the excited components. Such an opposite excitation enables the cancellation of the forces generated by deformation of the excited components and leads to the no change of reaction force from ground. Therefore, the deformation of the excited components can grow without takeoff of the lower body. Fig. 8 shows the schema of above discussion in the case of $N=2$.

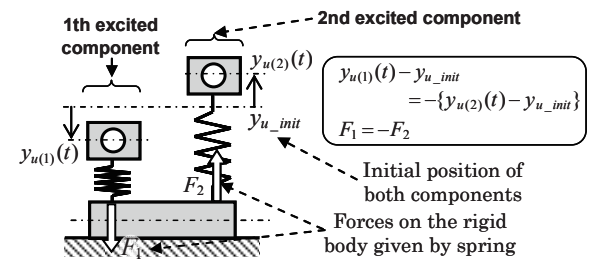

Fig. 8. Schema of cancellation of the forces generated by deformations due to resonant oscillation in case of $N=2$.

For the sake of convenience, the excitation procedure in this Subsection is called "opposite oscillation" in the later.

\subsection{Application of stiffness softening}

Suppose the same system as shown in Fig. 8. When the excited force is enough small, the displacements of the 1st and 2nd excited component for short time range are given by

$$
\begin{aligned}
& y_{u(1)}(t)=\Lambda \sin \omega_{f} t \\
& y_{u(2)}(t)=-\Lambda \sin \omega_{f} t
\end{aligned} .
$$

where $\Lambda$ is instantaneous amplitude. Consequently, the force on the rigid body given by the spring is represented as

$$
\begin{aligned}
& F_{(1)}=k \Lambda \sin \omega_{f} t \\
& F_{(2)}=-k \Lambda \sin \omega_{f} t
\end{aligned} .
$$

where $y_{u_{-} \text {init }}$ is negligible compared to $y_{u(1)}(t)$ and $y_{u(2)}(t)$. Suppose that the rigidity of the spring in the 2nd excited component decreases in small amount $\Delta k$ and consequently their natural frequency also decreases in small amount $\Delta \omega$. Then the resultant force by the springs are given as

$$
\begin{aligned}
F_{(1)}+F_{(2)} & =k \Lambda \sin \omega_{f} t-(k-\Delta k) \Lambda \sin \left(\omega_{f}-\Delta \omega\right) t \\
& =\Lambda \sqrt{\Delta k^{2}+(k-\Delta k)^{2} \Delta \omega^{2} t^{2}} \sin \left(\omega_{f} t+\phi\right)
\end{aligned} .
$$

Furthermore, suppose that $t$ is quite small, Eq.(19) can be expressed as

$$
F_{(1)}+F_{(2)} \approx \Lambda \Delta k \sin \left(\omega_{f} t+\phi\right)
$$

As is clear from comparison between Eq. (18) and Eq .(20), Eq. (20) indicate that the softening of the spring of one side functions as the generation of force by equivalent spring with the spring constant $\Delta k$ and the compressed displacement $\Lambda$. Therefore, even if stiffness changing rate is not enough high, the exciting method denoted in Subsection 4.1 can provide a larger force by the growth of oscillation amplitude. Therefore, the stiffness switching by piezoelectric element which shows small stiffness changing rate can be applied to the practical jumping mechanism.

\subsection{Numerical analyses and discussions}

According to the discussion in Subsection 3.2, stiffness can be softened by switching of the external capacitor from low capacitance to high capacitance. As discussed in Subsection 4.1 and 4.2, opposite resonant oscillation and stiffness softening yields a force by spring and it can be expected that 
the force lead to the jumping of the system. To validate the proposed jumping mechanism with proposed procedure, numerical analyses are carried out and the parameters for the analyses are shown in Table 2.

Table 2. Parameters for analysis in the case of stiffness softening.

\begin{tabular}{|l|l|}
\hline$N=10$ & $h_{b}=h_{p=} 0.5[\mathrm{~mm}]$ \\
\hline$L=0.10[\mathrm{~m}]$ & $m_{t}=m_{r}=5.0[\mathrm{~g}]$ \\
\hline$w_{b}=w_{p}=5.0[\mathrm{~mm}]$ & $g=9.81\left[\mathrm{~m} / \mathrm{s}^{2}\right]$ \\
\hline$\rho_{b}=2.7 \times 10^{3}\left[\mathrm{~kg} / \mathrm{m}^{3}\right]$ & $C_{E 1}=1.0 \times 10^{-9}[\mathrm{~F}]$ \\
\hline$\rho_{p}=7.7 \times 10^{3}\left[\mathrm{~kg} / \mathrm{m}^{3}\right]$ & $C_{E 1}=1.0 \times 10^{-6}[\mathrm{~F}]$ \\
\hline$E_{b}=7.0 \times 10^{10}[\mathrm{~Pa}]$ & $\varepsilon_{33}^{T}=5500$ \\
\hline$E_{p}=5.9 \times 10^{10}[\mathrm{~Pa}]$ & $d_{31}=-330 \times 10^{-12}[\mathrm{~m} / \mathrm{V}]$ \\
\hline
\end{tabular}

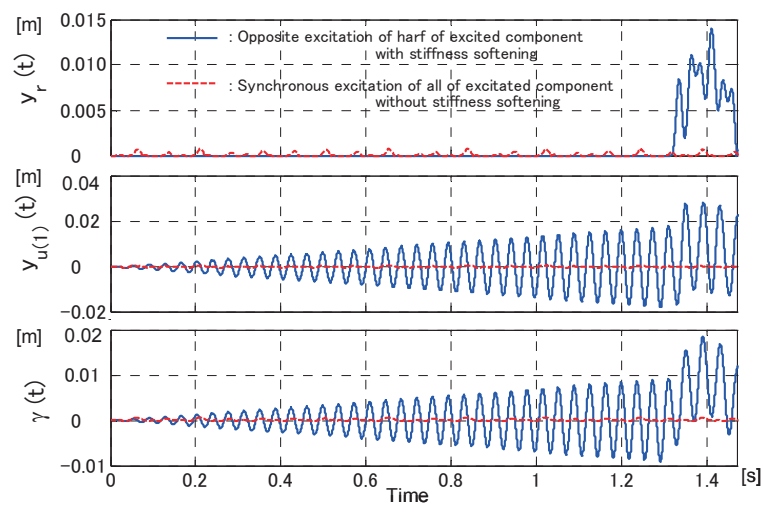

Fig. 9. Numerical analysis in case of stiffness softening.

Figures in Fig. 9 indicate same ones with those in Fig. 7. The blue solid line shows the results in the case of opposite excitation with stiffness softening. The Stiffness softening is carried out at $1.31[\mathrm{sec}$.$] when the half of excited component$ reaches the local maximum height. Red dashed line is the result in the case of synchronous excitation of all excited components without stiffness softening. As is clear from the results indicated by blue solid line, the rigid body does not take off from ground until stiffness is softened and the rigid body begins to take off from ground after stiffness softening at 1.31 [sec.]. Furthermore, achieved maximum height of rigid body and the center of mass are about $0.014[\mathrm{~m}]$ and $0.017[\mathrm{~m}]$ respectively. On the other hand the results indicated by red dashed line show that the system takes off at early time without enough storage of potential energy, which results in the low height of jumping. Therefore, it can be said that the proposed jumping mechanism and procedure is effective.

\section{Summary and Future Work}

\subsection{Summary}

- Jumping mechanism with stiffness hardening was proposed and its feature and problem were studied.

- Jumping mechanism with stiffness softening was proposed and numerical analysis for the model was carried out.

\subsection{Future work}

- Validation of proposed method by experiments.
- Optimization of the parameters to improve the hopping height of the proposed jumping mechanism.

- Construction of an objective valuation parameter in order to evaluate the amount of jumping height for development of practical application, e.g. :

\section{Jumping height}

$($ Rover mass $) \times($ Rover height $) \times($ Gravity acceleration $)$

- Horizontal locomotion method for practical application.

- Application of other functional materials instead of piezoelectric element.

- More strict theoretical analyses without approximation for the jumping mechanism with stiffness softening.

\section{Appendix}

$M_{11}=M_{G 1}=m_{r}+m_{t}+m_{f}, M_{12}=\frac{m_{f}}{L} \int_{0}^{L} \phi_{1} d x+m_{t} \phi_{1}(L)$,

$M_{22}=\frac{m_{f}}{L} \int_{0}^{L} \phi_{1}^{2} d x+m_{t} \phi_{1}(L)$,

$M_{G 2}=m_{t} \phi_{1}(L)+\frac{m_{f}}{L} \int_{0}^{L} \phi_{1} d x, m_{f}=\left(\rho_{b} A_{b}+\rho_{p} A_{p}\right) L$,

$K_{22}=\left(E_{b} I_{b}+\frac{I_{p}}{s_{11}^{E}}\right) \int_{0}^{L}\left(\frac{\partial^{2} \phi_{1}}{\partial x^{2}}\right)^{2} d x, \psi_{v}=\left(z-\frac{h_{b}}{2}\right) \frac{1}{h_{p}}$,

$\Theta=\int_{0}^{L} \int_{\frac{h_{b}}{2}}^{\frac{h_{b}}{2}+h_{p}} \frac{z w d_{31}}{s_{11}^{E}} \frac{\partial^{2} \phi_{1}}{\partial x^{2}} \frac{\partial \psi_{v}}{\partial z} d z d x$,

$C_{p}^{S}=\int_{0}^{L} \int_{\frac{h_{b}}{2}}^{\frac{h_{b}}{2}+h_{p}} w \varepsilon_{33}^{T}\left(\frac{\partial \psi_{v}}{\partial z}\right)^{2} d z d x$

\section{References}

1) Veverka, J. et al.: The landing of the NEAR-Shoemaker spacecraft on asteroid 433 Eros, Nature, 413 (6854), (2001), pp.390-393.

2) Rayman, M.D., Varghese, P, D.H. and Lehman, L.L.: Results from the Deep Space 1 technology validation mission, Acta Astronautica, 47, Issues 2-9, (2000), pp.475-487.

3) Fujiwara, A. et al.: The Rubble-Pile Asteroid Itokawa as Observed by Hayabusa, Science, 312, No.5778, (2006), pp.1330-1334.

4) Yoshimitsu, T., Nakatani, I. and Kubota, T.: New mobility system for small planetary body exploration, Proc. of IEEE Int. Conf. on Robotics and Automation, 2, (1999), pp.1404-1409.

5) Hougen, D. F. et al.: A Miniature Robotic System for Reconnaissance and Surveillance, Proc. of IEEE Int. Conf. on Robotics and Automation, (2000), pp.501-507.

6) Hosoda, K. et al.: Biped robot design powered by antagonistic pneumatic actuators for multi-modal locomotion, Robotics and Autonomous Systems, 56, (2008), pp.46-53.

7) Yamada, K. et al., Equivalent Mechanical and Electrical Models of the vibration Suppression System Using Piezoelectric Elements, Journal of the Japan Society of Mechanical Engineers, Series C, 73, No.730, (2007), pp.19-26.

8) Hagood, N.W. and Flotow, A.V.: Damping of structural vibrations with piezoelectric materials and passive electrical networks, Journal of Sound and Vibrations, 146-2, (1990), pp.243-268.

9) S.S. Rao: Mechanical vibrations forth edition, Pearson Education International, (2004).

10) Specification sheet of material characteristics about the lead zirconate titanate materials, Fuji Ceramics Corporation. 